\title{
Competências da equipe de enfermagem frente aos cuidados paliativos em pacientes
}

\section{com câncer de colo de útero}

Skills of the nursing team regarding palliative care in patients with cervical cancer

Habilidades del equipo de enfermería en cuidados paliativos en pacientes con cáncer de cuello uterino

\section{Resumo}

Realizar uma busca na literatura sobre as principais competências da equipe de enfermagem mediante aos cuidados paliativos em pacientes com câncer de colo de útero, abordando em conjunto suas principais condutas e suas dificuldades. Trata-se de um estudo de origem descritivo, exploratório, com abordagem qualitativa, utilizando a Revisão Integrativa da Literatura (RIL) como técnica. Para coleta de dados, foram utilizadas as bases de dados disponíveis e indexadas dentro da Biblioteca Virtual de Saúde: SCIELO, LILACS, BDENF e MEDLINE, mediante a junção de três Descritores em Saúde, cruzados com o operador booleano "AND": Cuidados Paliativos AND Neoplasias do Colo do Útero AND Cuidados de Enfermagem. Sendo encontrados 236 artigos na totalidade, após aplicações dos critérios de inclusão e exclusão, 13 artigos foram selecionados. As pesquisas abordaram sobre a importância do enfermeiro mediante a orientação dos cuidados paliativos, onde enfatizam que a comunicação correta é primordial para que seja possível ensiná-las a lidar com as alterações, reforçando diálogos para amenizar o isolamento e o medo, reforçando a importância do apoio familiar. Mediante as diversas dificuldades apresentadas por esses profissionais, é importante uma autorreflexão sobre essa temática, contribuindo assim não somente para prática assistencial, mas também para melhor qualidade de vida da família e assistidos.

Palavras-chave: Cuidados paliativos; Neoplasias do colo do útero; Cuidados de enfermagem.

\begin{abstract}
Conduct a literature search on the main competencies of the nursing team through palliative care in patients with cervical cancer, jointly addressing their main conducts and difficulties. This is a descriptive, exploratory study, with a qualitative approach, using the Integrative Literature Review (RIL) as a technique. For data collection, the databases available and indexed within the Virtual Health Library were used: SCIELO, LILACS, BDENF and MEDLINE, by joining three Health Descriptors, crossed with the Boolean operator "AND": Palliative Care AND Cervical Neoplasms AND Nursing Care. 236 articles were found in total, after applying the inclusion and exclusion criteria, 13 articles were selected. The researches addressed the importance of the nurse through the guidance of palliative care, emphasizing that correct communication is essential so that it is possible to teach them to deal with changes, reinforcing dialogues so that it is possible to alleviate isolation and fear, reinforcing the importance of family support. Due to the various difficulties presented by these professionals, a self-reflection on this theme is extremely important, thus contributing not only to care practice, but also to a better quality of life for the family and those assisted.
\end{abstract}

Keywords: Palliative care; Cervical neoplasms; Nursing care. 


\begin{abstract}
Resumen
Realizar una búsqueda bibliográfica sobre las principales competencias del equipo de enfermería a través de los cuidados paliativos en pacientes con cáncer cervicouterino, abordando de forma conjunta sus principales conductas y dificultades. Se trata de un estudio descriptivo, exploratorio, con enfoque cualitativo, utilizando como técnica la Revisión Integrativa de Literatura (RIL). Para la recolección de datos se utilizaron las bases de datos disponibles e indexadas dentro de la Biblioteca Virtual en Salud: SCIELO, LILACS, BDENF y MEDLINE, uniendo tres Descriptores de Salud, cruzados con el operador booleano "Y": Cuidados Paliativos Y Neoplasias Cervicales Y Cuidados de Enfermería. Se encontraron 236 artículos en total, luego de aplicar los criterios de inclusión y exclusión, se seleccionaron 13 artículos. Las investigaciones abordaron la importancia del enfermero a través de la orientación de los cuidados paliativos, enfatizando que la comunicación correcta es fundamental para que sea posible enseñarles a afrontar los cambios, reforzando los diálogos para que sea posible aliviar el aislamiento y el miedo, reforzando la importancia de apoyo familiar. Debido a las diversas dificultades que presentan estos profesionales, la autorreflexión sobre este tema es de suma importancia, contribuyendo así no solo a la práctica asistencial, sino también a una mejor calidad de vida de la familia y los asistidos.
\end{abstract}

Palabras clave: Cuidados paliativos; Neoplasias cervicales; Cuidado de enfermera.

\title{
1. Introdução
}

Segundo o Instituto Nacional do Câncer (INCA), o câncer é descrito como o crescimento desordenado das células, podendo ou não, invadir outros tipos de tecidos adjacentes ou órgãos a distância. Essas células possuem a capacidade de se dividirem rapidamente e tendem a ser agressivas, formando tumores em diferentes partes do corpo. Atualmente, existem mais de 100 tipos diferentes de tumores, destacando-se o câncer de colo de útero, onde no Brasil, estima-se que aproximadamente a cada 100 mil mulheres 15,43\% por ano poderão desenvolver essa doença (Lopes, 2019).

O Câncer de Colo de Útero (CCU) é um tumor (maligno ou benigno) que se desenvolve a partir de lesões (percursoras) que ocorrem no colo do útero, localizado no fundo da vagina, sendo decorrente de alterações celulares com disseminação das células anormais de forma progressiva e gradativa, considerando-se como uma doença crônico-degenerativa, devido seu alto índice de morbidade (Roman, 2015).

Segundo o Organização Mundial de Saúde (OMS), o CCU é a terceira maior causa de mortalidade para as mulheres. Mediante a isso, existem vários fatores que podem contribuir para o alargamento do Câncer Cérvico-Uterino, como: relação sexual precoce, relação com mais de um parceiro, menstruação precoce ou tardia, infecção pelo Papilomavírus Humano (HPV), infecção genital, condição socioeconômica baixa, entre outros (Oms, 2018).

O CCU corresponde a um grave problema de saúde pública no Brasil, sendo uma doença grave que evolui lentamente, provocando um aumento nos números dos casos, principalmente em mulher que apresentam baixa condição social e econômica. Frente a isso, no Brasil entre os anos de 2016 a 2017 foram diagnosticados mais de 16.340 casos novos de Câncer Uterino segundo o INCA (Costa, 2017).

A infecção pelo HPV é a principal causa associada ao desenvolvimento do CCU. Por meio disso, a Agência Internacional para Pesquisa sobre o Câncer (AIPC), enfatiza que existem mais de 13 tipos de HPV (oncogênicos), sendo o HPV (16 e 18), onde causam mais de 70\% dos cânceres dos CCU e lesões pré-cancerosas. Essas lesões são classificadas como neoplasia intraepitelial celular (NIC) I e II, que são detectadas por meio do exame citopatológico (Guerreiro, 2017).

O diagnóstico do CCU é feito por meio do exame coletado na atenção básica de saúde, sendo realizado principalmente pelo enfermeiro. Entretanto, existem outros métodos já preconizados, como o processo de rastreamento, onde é solicitado uma busca ativa afim de detectar precocemente qualquer tipo de alteração celular presente (Ferraz, 2019).

Mediante a isso, o tratamento é determinado de acordo com o tipo de câncer, existindo várias estratégias terapêuticas frente ao CCU, como: biopsia a laser, crioterapia, histerectomia, quimioterapia e até mesmo a radioterapia. Por outro lado, algumas pesquisas ressaltam que lesões de baixo grau em mulheres com idade menor de 30 anos, podem ocorrer regressão espontânea (Silva, 2018). 
Através da atenção primaria é que as mulheres são orientadas pela equipe de saúde sobre a prevenção do CCU, através de medidas importantes como: vacinação, preventivo e, principalmente, as medidas de autocuidado sobre as vias de transmissão. Frente a isso, o profissional de enfermagem possui inúmeras atribuições mediante a essa patologia, tanto em ações voltadas para orientação e prevenção, assim como também a ações relacionadas a prestação de cuidados paliativos a pacientes com CCU (Gomes, 2017).

Diante disso, é de extrema importância a capacitação correta desses profissionais com a finalidade de evitar acidentes errôneos a essas mulheres, dispondo sempre de um atendimento focado no paciente como pessoa humana, não como uma patologia que precisa ser tratada, através de uma assistência segura e humanizada, prestando os principais cuidados para essas pacientes acometidas por essa complicação (Costa, 2021).

Diante das informações expostas, o presente estudo justifica-se devido ao CCU ser um dos principais tipos de cânceres que mais matam mulheres do mundo. Logo, ainda é bastante alto o índice de mulheres acometidas por essa complicação, devido ao alcance pequeno das campanhas do Sistema Único de Saúde (SUS) nas unidades que abrangem o Programa de Assistência Integral à Saúde da Mulher (Costa, 2016).

Por meio disso, é importante destacar que quanto mais cedo for a detecção (precoce ou não) do CCU, maiores são as chances de cura, iniciando o tratamento o mais rápido possível. $\mathrm{O}$ apoio da família também é primordial assim como a assistência de médicos e enfermeiros nesse processo que envolve desde o acolhimento até a introdução de cuidados paliativos frente ao tratamento da patologia (Silva et al, 2016).

Desse modo, justifica-se a importância da temática para os acadêmicos e profissionais de enfermagem pela necessidade do conhecimento e contribuição para discussão e debate sobre o assunto, assim como a melhora da prática assistencial, uma vez que, profissionais de enfermagem precisam em certos momentos de a profissão atuar como um psicólogo, ou seja, saber ouvir e dar conselhos, e principalmente acompanhar a paciente durante essa difícil jornada (Moutinho, 2017). Frente a isso, a seguinte pesquisa tem por objetivo realizar uma busca na literatura sobre as principais competências da equipe de enfermagem mediante aos cuidados paliativos em pacientes com câncer de colo de útero, abordando em conjunto suas principais condutas e suas dificuldades.

\section{Metodologia}

Trata-se de uma Revisão Integrativa da Literatura (RIL), que é um método que proporciona conhecimento e resultados na prática, tornando possível reunir conhecimentos publicados anteriormente durante anos distintos com diferentes abordagens metodológicas, incorporando em conjunto conceitos, revisão de teorias, e evidências e análise de problemas (Botelho et al., 2011).

Para coleta de dados, foram utilizadas as bases de dados disponíveis e indexadas dentro da Biblioteca Virtual de Saúde (BVS): Biblioteca Eletrônica Científica Online (SCIELO), Literatura Latino-Americana e do Caribe em Ciências da Saúde (LILACS), Base de Dados Bibliográficas Especializada na área de Enfermagem (BDENF), em conjunto com o Sistema Online de Busca, Análise de Literatura Médica (MEDLINE) e Índice Bibliográfico Español en Ciencias de la Salud (IBECS), através da junção de três Descritores em Saúde (DeCS) cruzados com o operador booleano "AND": "Cuidados Paliativos, Neoplasias do Colo do Útero, Cuidados de Enfermagem". 
Tabela 1: Bases de dados e quantidade de artigos selecionados.

\begin{tabular}{|c|c|c|c|}
\hline \multicolumn{5}{|c|}{ Biblioteca Virtual de Saúde } \\
\hline $\mathrm{N}^{\circ}$ & Bases de dados & Total & Selecionados \\
\hline 1 & LILACS & 58 & 4 \\
\hline 2 & BDENF & 43 & 3 \\
\hline 3 & MEDLINE & 134 & 2 \\
\hline 4 & SCIELO & 4 & 4 \\
\hline 5 & IBECS & 10 & 0 \\
\hline
\end{tabular}

Fonte: Autores (2021).

A pesquisa apresenta os seguintes critérios de inclusão: artigos que foram publicados dentro dos idiomas português e inglês, dentro dos anos (2016 a 2021) que estivessem disponíveis de forma gratuita na internet. Referente aos critérios de inelegibilidade, foram descartadas monografias, artigos indisponíveis gratuitamente, dissertações de mestrado e teses de doutorado, além do descarte de publicações anteriores ao no de 2016 e fora dos idiomas estabelecidos nos critérios de inclusão (Figura 1).

Figura 1: Fluxograma das etapas de seleção dos artigos para revisão.

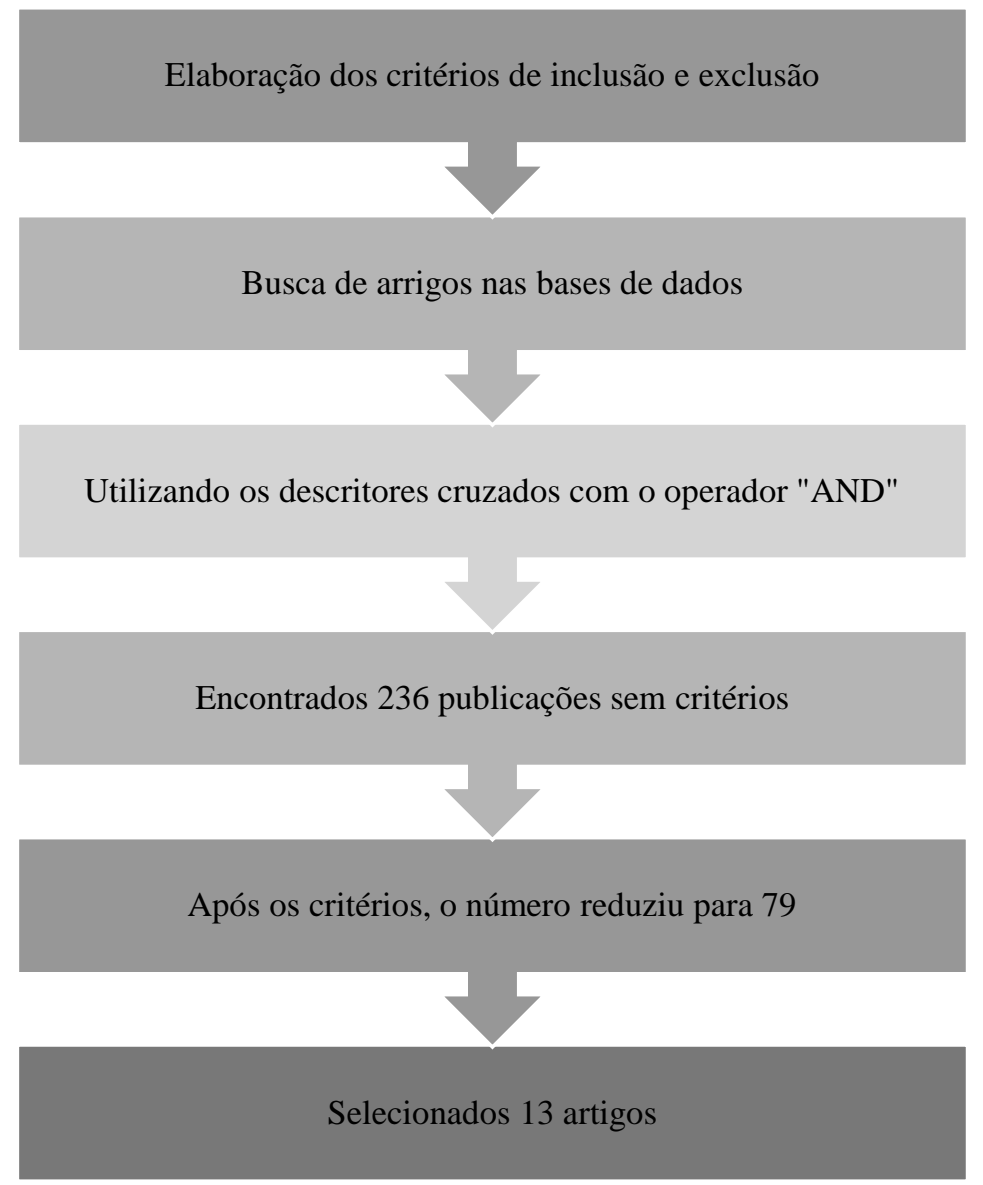

Fonte: Autores (2021).

\section{Resultados}

Utilizando os seguintes DeCS: “Cuidados Paliativos, Neoplasias do Colo do Útero. Cuidados de Enfermagem” foram encontrados 236 artigos. Ao adicionar os critérios de inclusão e exclusão o número reduziu para 79. Após análise e leitura das 
publicações, 13 artigos direcionados ao objetivo do estudo foram selecionados. Para que as publicações selecionadas fossem compreendidas da melhor forma possível, será apresentado uma síntese das publicações conforme autor, ano, título, estudo, resultados e publicação, facilitando assim na análise dos artigos que compuseram essa revisão integrativa (Quadro 1).

Quadro 1: Artigos selecionados para compor esta revisão.

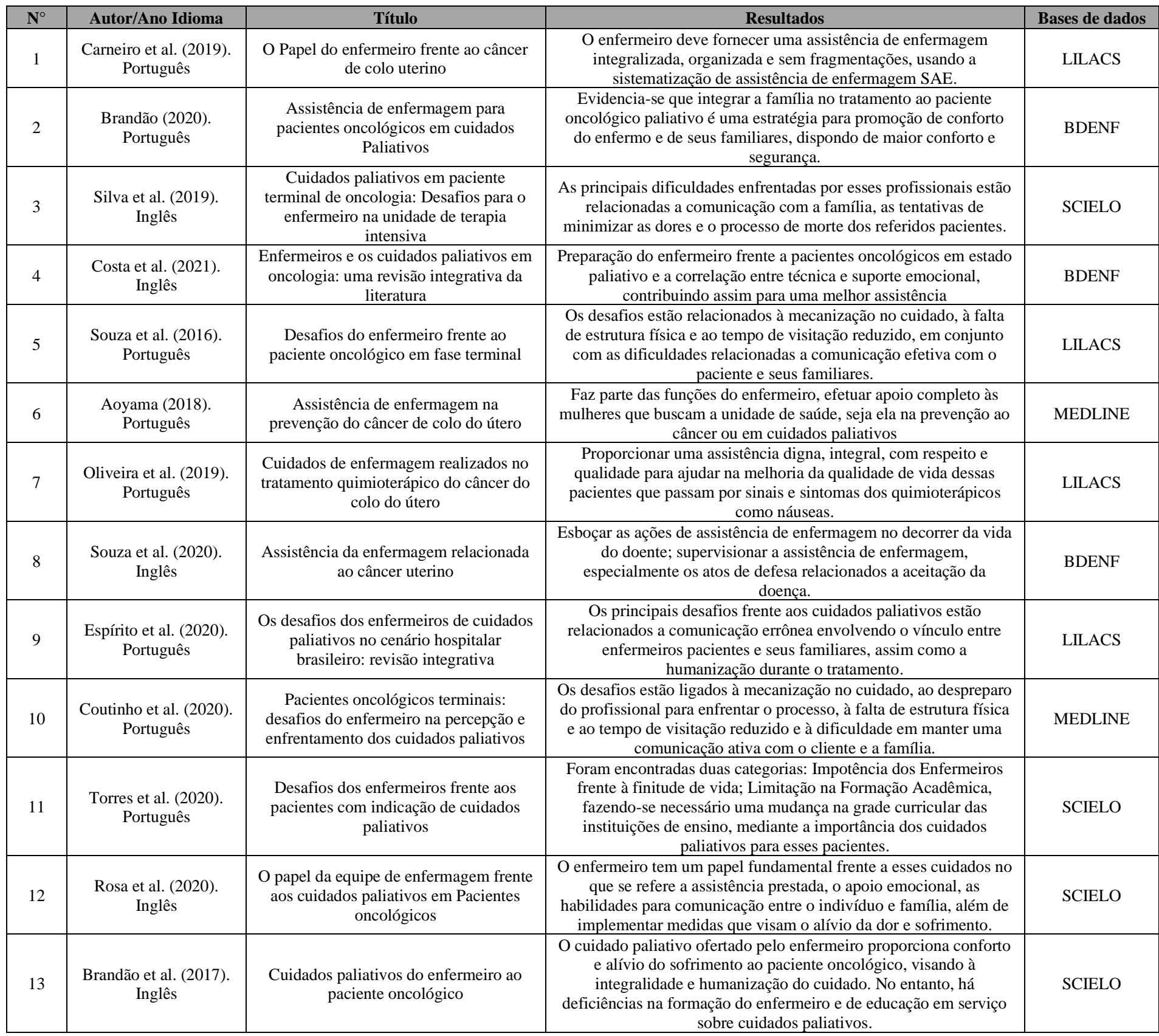

Fonte: Autores (2021).

\section{Discussão}

Após a análise dos artigos selecionados, as discussões dos resultados encontrados foram abordadas de duas formas distintas, porém intercaladas a fim de promover aos leitores um melhor entendimento referente ao tema (Brandão et al. 2020). Abordou-se primeiramente sobre as condutas da equipe de enfermagem frente pacientes terminais com câncer de colo do útero, logo após, os desafios apresentados pela equipe de enfermagem frente as condutas direcionadas a essas pacientes. 


\subsection{Condutas da equipe de enfermagem frente pacientes terminais com câncer de colo do útero}

Segundo Carneiro et al. (2019), pacientes que foram diagnosticadas com CCU em estágio terminal e estão sob cuidados paliativos, passam por diversas situações que podem influenciar diretamente na sua qualidade de vida, como: ansiedade, debilitação, distúrbio de autoimagem, incontinências fisiológicas, e principalmente o medo da morte.

Frente a isso, Brandão et al. (2020) aborda que a equipe de enfermagem possui um papel significativo diante dos cuidados prestados a essas mulheres, contribuindo diretamente na melhora da qualidade de vida em todos os aspectos, onde segundo a OMS as condutas devem ser direcionadas para questões que envolvam fatores físicos, psicológicos e sociais, levandose em consideração a percepção da paciente em relação a patologia que à acomete.

Em consenso, Aoyana (2018) e Costa et al. (2021), abordam sobre a importância do enfermeiro na orientação dos cuidados paliativos, onde enfatizam que a comunicação correta é primordial para que seja possível ensiná-las a lidar com possíveis alterações, reforçando diálogos para que seja possível amenizar o isolamento e o medo, reforçando a importância do apoio familiar.

Outro aspecto relevante é considerar e utilizar as crenças para prestar os cuidados, usando como aliada ao bem-estar, contribuindo para que as condutas assistenciais da equipe de enfermagem sejam realizadas de maneira integralizada, observando o paciente de maneira holística, em conjunto com a Sistematização da Assistência de Enfermagem (SAE) para embasamento, intervenções e avaliação do resultado esperado de modo correto (Oliveira et al., 2019).

Brandão et al. (2020), cita que a assistência paliativa não vislumbra a cura, mas sim minimizar o sofrimento através do alívio da dor. Com isso, o enfermeiro deve possuir total conhecimento do quadro clínico que o paciente apresenta em conjunto com as abordagens terapêuticas para os sintomas em destaque, para que assim possa ser possível a orientação correta aos seus familiares de como proceder, dispondo de apoio sempre que for preciso.

Segundo Markus et al. (2017) as ações assistenciais estão direcionadas ao controle e alívio da dor, em conjuntos com os demais sintomas, proporcionando uma melhor qualidade de vida para o paciente. Onde segundo Brandão et al. (2020), é de responsabilidade do enfermeiro otimizar o cuidado, exercendo uma ótima avaliação dos sintomas e suas intensidades, na forma de minimizar as complicações impostas pelo agravo da patologia oncológica avançada.

Corroborando com a pesquisa de Brandão et al. (2020), Abreu et al. (2018) abordam que a equipe de enfermagem possui papel extremamente válido no encorajamento dos cuidadores do paciente oncológico em conjunto com a identificação e minoração dos fatores estressores, para que seja possível a potencialização dos resultados a serem obtidos, por meio do fortalecimento geral dos cuidadores.

Acrescido a isso, Wakiuchi e Sales (2018), enfatizam que a equipe de enfermagem deve estar preparada para lidar diretamente com os sentimentos e angústia dos pacientes que se deparam com a incurabilidade do CCU. Nesse sentido, Oliveira et al. (2017), firmam que os desafios e sofrimentos de cuidar de alguém que não apresente possibilidades de cura terapêutica, torna possível que os cuidadores desenvolvam autoconhecimento, aflorando as necessidades de buscar determinadas maneiras de minimizar o sofrimento do paciente.

Souza et al. (2020) e Oliveira et al. (2019), firmam que gerenciar o cuidado de enfermagem ao paciente com câncer em estágio terminal é uma ação que surge a partir da valorização do indivíduo no seu contexto de vida, buscando atender as necessidades dos cuidados que são impostos pela doença ou até mesmo pela própria terapêutica instalada, visando o alcance de resultados positivos, incluindo o atendimento de qualidade ao paciente e seus familiares.

Segundo Carneiro et al. (2016) alguns cuidados frente ao leito da paciente em estado terminal são introduzidos, com destaque para avaliação do estado nutricional, que é voltado diretamente com o aumento de morbimortalidade e infecções, dificultando assim a resposta ao tratamento e acompanhamento da avaliação contínua a essas pacientes. Frente a isso, Brandão 
et al. (2020) citam em sua pesquisa sobre a importância da adesão terapêutica, onde o enfermeiro tem o papel de orientar a paciente e seus familiares sobre o tratamento, saber ouvir seus sentimentos, e reforçar sobre diálogos amenizem seus medos.

Acrescido a isso, Costa et al. (2021), abordam em sua pesquisa sobre ações frente ao leito que possibilitem o alívio da dor, destacando a importância do conhecimento do enfermeiro total do quadro clínico da paciente, em conjunto com as abordagens metodológicas que estão sendo realizadas para amenizar os sintomas, e orientar a família de como proceder, dispondo de apoio sempre que precisarem.

Adicionalmente, Oliveira et al. (2020) citam em sobre cuidados realizados no leito que contribuem para a manutenção da saúde e bem-estar da paciente, destacando sobre a ingestão de alimentos em porções pequenas, frios ou em temperatura ambiente, quando a mesma apresenta náuseas e vômitos frente a realização do tratamento, em conjunto com o uso de medicamentos antiemético, evitando alimentos doces, gordurosos ou com odor forte, diminuindo assim as chances de anorexia.

\subsection{Dificuldades encontradas pela equipe de enfermagem frente aos cuidados com pacientes com câncer de colo de útero}

Segundo Espirito et al. (2020), o enfermeiro enfrenta inúmeros desafios frente a introdução dos cuidados paliativos em pacientes com câncer de colo de útero em estágio terminal, como o processo de mecanização, que ocorre quando o profissional se preocupa apenas com a técnica, estando despreparado para prestar a assistência ao paciente em estágio terminal como um todo. Acrescido a isso, Oliveira et al. (2017), abordam em sua pesquisa sobre as dificuldades em controlar seus sentimentos com os do paciente, com a possível morte dele, em conjunto com a falta de estrutura física da unidade hospitalar, que dificulta o processo do cuidado.

Outro aspecto importante segundo Silva et al. (2019), é a internação do paciente terminal, onde algumas questões são abordadas com frequência nesse processo, como: a ausência de acomodações adequadas, desconforto para os familiares durante as visitas, já que as mesmas tem por objetivo prestar apoio emocional ao paciente, gerando o reestabelecimento da sua saúde.

Adicionalmente, Souza et al. (2016) e Silva et al. (2019) enfatizam em sua pesquisa no que tange a comunicação (verbal ou não) a principal dificuldade apresentada pelos profissionais de enfermagem refere-se as relações interpessoais, devido a demanda demasiada dos pacientes, acrescido a carência das informações pelos acompanhantes e familiares do paciente nas tentativas de minimizar as dores e o processo de morte dos referidos pacientes.

Segundo torres et al. (2020), a comunicação é um processo indispensável para que as ações de independência funcional do indivíduo em fase terminal sejam preservadas, uma vez que a mesma auxilia diretamente no descobrimento do senso de controle, contribuindo assim no processo de tomada de decisões, melhorando o relacionamento interpessoal entre os profissionais e os pacientes, contribuindo para o aumento da confiança e segurança aos assistidos.

Coutinho et al. (2020), abordam em sua pesquisa que embora o avanço das tecnologias venha crescendo a cada dia com relação as doenças, muitos profissionais ainda enfrentam muitos desafios na assistência ao paciente que se encontra em fase terminal da doença, percebendo a importância da introdução de processos humanizados, enfatizando a importância da introdução dessa temática dentro da grade curricular da graduação, para que assim seja possível a reflexão sobre a importância da prestação de um cuidado holístico mediante a qualidade de vida desse paciente.

\section{Considerações Finais}

Diante do exposto, no que tange a importância desses profissionais para prática clínica e assistencial na introdução dos cuidados paliativos, torna-se de suma importância o preparo científico e correto, baseado em evidências desses profissionais, para que assim seja possível dispor de cuidados de qualidade às pacientes que se encontram em estágio terminal. Logo, mediante as diversas dificuldades apresentadas por esses profissionais, é extremamente importante uma autorreflexão sobre essa temática, 
contribuindo assim não somente para prática assistencial, mas também para uma melhor qualidade de vida da paciente, família e assistidos. Diante disso, espera-se que a seguinte pesquisa possa servir de subsídio para futuras pesquisas envolvendo a atual temática, contribuindo assim para o bem-estar físico e mental de todos os envolvidos.

\section{Referências}

Amaral, A. F., Araújo, E. S., Magalhães, J. C., Silveira, É. A., Tavares, S. B. D. N., \& Amaral, R. G. (2014). Impacto da capacitação dos profissionais de saúde sobre o rastreamento do câncer do colo do útero em unidades básicas de saúde. Revista Brasileira de Ginecologia e Obstetrícia, 36, $182-187$.

Aoyama, E. A., da Silva Pimentel, A., de Andrade, J. S., Daniel, W. V., de Souza, R. A. G., \& Lemos, L. R. (2019). Assistência de enfermagem na prevenção do câncer de colo do útero. Brazilian Journal of Health Review, 2(1), 162-170.

Amaral, M. S., Gonçalves, A. G., \& Silveira, L. C. G. (2017). Prevenção do câncer de colo de útero: a atuação do profissional enfermeiro nas unidades básicas de saúde. Revista Científica FacMais, 8(1), 198-223.

Batista, R. C. L. (2015). Papel da enfermagem na prevenção, diagnóstico e tratamento do câncer de colo uterino: uma revisão integrativa.

Brandão, M. C. P., Sampaio, K. C. P., Mochizuki, A. B., \& Santos, V. C. (2017). Cuidados paliativos do enfermeiro ao paciente oncológico. Revista Brasileira de Saúde Funcional, 1(2), 76-76.

Brandão, M. L. (2020). Assistência de enfermagem para pacientes oncológicos em cuidados paliativos: importância da interação familiar no tratamento. Caderno de Graduação-Ciências Biológicas e da Saúde-UNIT-SERGIPE, 6(1), 175

Botelho, L. L. R., de Almeida Cunha, C. C., \& Macedo, M. (2011). O método da revisão integrativa nos estudos organizacionais. Gestão e sociedade, 5(11), 121-136.

Carneiro, C. P. F., Pereira, D. M., Pereira, A. T., Santos, G. A. S., de Moraes, F. A. D. S., \& Duarte, R. D. F. R. (2019). O Papel do enfermeiro frente ao câncer de colo uterino. Revista Eletrônica Acervo Saúde, (35), e1362-e1362.

Costa, J. O., dos Santos, F., Lohmann, P. M., \& Bernardes, C. (2021). Enfermeiros e os cuidados paliativos em oncologia: uma revisão integrativa da literatura. Research, Society and Development, 10 (3), e35210310642-e35210310642.

Costa, A. M. (2016). O Programa de Assistência Integral a Saúde da Mulher: anotações para um debate. Anais, 1133-1143.

Cunha, E. S. (2016). Assistência de enfermagem na prevenção do câncer de colo uterino. FACIDER-Revista Científica, (09).

Duarte, D. D. A. P., \& Bustamante-Teixeira, M. T. (2018). Social Iniquity and Mortality Related to Breast and Cervical Cancers: An Integrative Review/Iniquidade Social e Mortalidade por Câncer de Mama e Colo do Útero: Uma Revisão Integrativa. Revista de Pesquisa Cuidado é Fundamental Online, $10(3), 877-888$

Ferraz, E. T. R., de Jesus, M. E. F., \& Leite, R. N. Q. (2019). Ações educativas: papel da (o) enfermeira (o) na prevenção do câncer do colo do útero. Brazilian Journal of Development, 5(10), 21083-21093.

Guerreiro, J. A., Salles, F. V., Villela, I. R., de Miranda, L. F., \& Kusma, S. Z. (2017). Lesões precursoras e malignas de colo uterino-incidência conforme a idade. Revista Médica da UFPR, 4(2), 61-66.

Gomes, L. C. D. S. et al. (2017). Conhecimento de mulheres sobre a prevenção do câncer de colo do útero: uma revisão integrativa. Revista UNINGÁ Review, $30(2)$

Lopes, V. A. S., \& Ribeiro, J. M. (2019). Fatores limitadores e facilitadores para o controle do câncer de colo de útero: uma revisão de literatura. Ciência \& Saúde Coletiva, 24, 3431-3442.

Machado, H. S., de Souza, M. C., \& da Cunha Gonçalves, S. J. (2017). Câncer de colo de útero: análise Epidemiológica e Citopatológica no município de Vassouras-RJ. Revista Pró-UniverSUS, 8(1)

Medeiros-Verzaro, P., \& Hélia de Lima Sardinha, A. (2020). Caracterização sociodemográfica e clínica de idosas com câncer do colo do útero. Revista de Salud Pública, 20, 718-724.

Rosa, N. M., de Matos Silva, R. K. P., Moreira, V. M., de Souza Silva, L. G., da Costa, R. S. L., \& Machado, M. P. (2021). O papel da equipe de enfermagem frente aos cuidados paliativos em pacientes oncológicos. DêCiência em Foco, 4(2), 82-93.

Souza, S. A. N., Souto, G. R., \& dos Santos, W. L. (2020). Assistência da enfermagem relacionada ao câncer uterino. Revista JRG De Estudos Acadêmicos, 3(6), 04-11.

Silva, L. R., Almeida, C. A. P. L., de Moura Sá, G. G., Moura, L. K. B., \& Araújo, E. T. H. (2017). Educação em saúde como estratégia de prevenção do câncer do colo do útero: revisão integrativa. Revista Prevenção de Infecção e Saúde, 3(4).

Soares, C. B., Hoga, L. A. K., Peduzzi, M., Sangaleti, C., Yonekura, T., \& Silva, D. R. A. D. (2014). Revisão integrativa: conceitos e métodos utilizados na enfermagem. Revista da Escola de Enfermagem da USP, 4, 335-345.

Silva, M. L., Nunes, J. S. S., de Oliveira, K. S., \& Leite, T. A. S. (2020). Conhecimento de mulheres sobre câncer de colo do útero: Uma revisão integrativa. Brazilian Journal of Health Review, 3(4), 7263-7275. 
Research, Society and Development, v. 10, n. 16, e379101623981, 2021

(CC BY 4.0) | ISSN 2525-3409 | DOI: http://dx.doi.org/10.33448/rsd-v10i16.23981

Silva, M. R. F. D., Braga, J. P. R., Moura, J. F. D. P., \& Lima, J. T. D. O. (2016). Continuidade Assistencial a mulheres com câncer de colo de útero em redes de atenção à saúde: estudo de caso, Pernambuco. Saúde em Debate, 40, 107-119.

Tsuchiya, C. T., Lawrence, T., Klen, M. S., Fernandes, R. A., \& Alves, M. R. (2017). O câncer de colo do útero no Brasil: uma retrospectiva sobre as políticas públicas voltadas à saúde da mulher. JBES: Brazilian Journal of Health Economics/Jornal Brasileiro de Economia da Saúde, 9(1).

Torres, C. M. G., dos Santos Nunes, S., Piovesan-Rosanelli, C. L. S., Conterato, D. M., \& Dias, C. F. C. Desafios dos enfermeiros frente aos pacientes com indicação de cuidados paliativos. Varia Scientia-Ciências da Saúde, 6(2), 137-147.

Viana, M. R. P., Moura, M. E. B., Nunes, B. M. V. T., de Sousa Monteiro, C. F., \& Lago, E. C. (2013). Formação do enfermeiro para a prevenção do câncer de colo uterino [Nursing education for prevention of cervical câncer]. Revista enfermagem UERJ, 21(5), 624-630. 